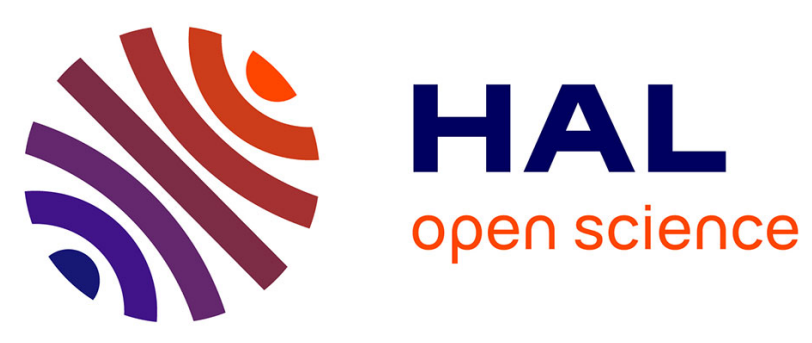

\title{
Interactive Training System for Interventional Electrocardiology Procedures
}

\author{
H Talbot, F Spadoni, Christian Duriez, M Sermesant, Stéphane Cotin, Hervé \\ Delingette
}

\section{- To cite this version:}

H Talbot, F Spadoni, Christian Duriez, M Sermesant, Stéphane Cotin, et al.. Interactive Training System for Interventional Electrocardiology Procedures. 6th International Symposium on Biomedical Simulation - ISBMS 2014, Oct 2014, Strabsourg, France. pp.11 - 19, 10.1007/978-3-319-12057-7_2 . hal-01078209

\section{HAL Id: hal-01078209 \\ https://inria.hal.science/hal-01078209}

Submitted on 25 Aug 2015

HAL is a multi-disciplinary open access archive for the deposit and dissemination of scientific research documents, whether they are published or not. The documents may come from teaching and research institutions in France or abroad, or from public or private research centers.
L'archive ouverte pluridisciplinaire HAL, est destinée au dépôt et à la diffusion de documents scientifiques de niveau recherche, publiés ou non, émanant des établissements d'enseignement et de recherche français ou étrangers, des laboratoires publics ou privés. 


\title{
Interactive Training System for Interventional Electrocardiology Procedures
}

\author{
H. Talbot ${ }^{1,2}$, F. Spadoni ${ }^{2}$, C. Duriez ${ }^{1}$, M. Sermesant ${ }^{2}$, S. Cotin ${ }^{1}$, and \\ H. Delingette ${ }^{2}$ \\ 1 Shacra Team, Inria Lille - North Europe, France \\ 2 Asclepios Team, Inria Sophia Antipolis - Méditerranée, France
}

\begin{abstract}
Recent progress in cardiac catheterization and devices allowed to develop new therapies for severe cardiac diseases like arrhythmias and heart failure. The skills required for such interventions are still very challenging to learn, and typically acquired over several years. Virtual reality simulators can reduce this burden by allowing to practice such procedures without consequences on patients. In this paper, we propose the first training system dedicated to cardiac electrophysiology, including pacing and ablation procedures. Our framework involves an efficient GPU-based electrophysiological model. Thanks to an innovative multithreading approach, we reach high computational performances that allow to account for user interactions in real-time. Based on a scenario of cardiac arrhythmia, we demonstrate the ability of the user-guided simulator to navigate inside vessels and cardiac cavities with a catheter and to reproduce an ablation procedure involving: extra-cellular potential measurements, endocardial surface reconstruction, electrophysiology mapping, radio-frequency ( $\mathrm{RF}$ ) ablation, as well as electrical stimulation. This works is a step towards computerized medical learning curriculum.
\end{abstract}

\section{Introduction}

Among all cardiovascular diseases, cardiac arrhythmia and heart failure are lifethreatening pathologies. Cardiac arrhythmia consists in an abnormal electrical activity in the myocardium (heart walls). Depending on the pathology, different therapies are pursued. RF ablation is preferred for ventricular tachycardia. In this paper, we only consider ventricular extrasystole, i.e. ventricular tachycardia caused by ectopic foci. An ectopic focus is an abnormal pacemaker area (outside of the sinoatrial node) that initiates abnormal self-generated beats. Such pathologies can occur upon changes in the heart structure following a coronary artery disease or as chronic consequences of hypertension, diabetes or cardiomyopathy, as stated in [9].

Considering an ectopic focus located inside the right ventricle (RV), the procedure first consists in inserting catheters from the femoral vein up to the RV under fluoroscopic imaging. Once inside the ventricle, an electrophysiology mapping is performed by exploring the endocardial surface with catheters to map 
the activation patterns. These patterns allow to locate the ectopic focus responsible for the arrhythmia. Each pathological region found by electrophysiology mapping will eventually be ablated using RF: heating the cardiac tissue next to the ectopic focus leads to cellular death, thus suppressing the related abnormal beats.

Until now, residents in cardiology train on patients by separately learning each step of the procedure under the supervision of a senior cardiologist. In order to shorten the training period and to allow a virtual training on complex patient cases, we propose a training system for interventional cardiology based on the simulation of electrophysiology.

Previous work Previous research projects already led to training simulators in cardiology as for instance detailed in [4]. The most recent simulator detailed in [3] focuses on intra-ventricular navigation. Main contributions of [3] include the reproduction of the conditions for the slip and nonslip interaction of the catheter. Authors present a qualitative analysis of the catheterization training using experimental data on a porcine left ventricle, as well as an user evaluation. Few training systems succeeded to be commercialized such as Cathi from Siemens, VIST (Vascular Intervention Simulation Trainer) from Mentice, Simantha from SimSuite, CathLabVR from CAE HealthCare and Angio Mentor from Simbionix. All these simulators are mostly focusing on the endovascular navigation including pre-recorded electrocardiograms (ECG), but none of these simulators neither includes a biophysical modeling of the cardiac electrophysiology, nor the interaction with the electrophysiology model.

Simulating the human cardiac electrophysiology is a wide field of research. However, only recent work $[11,12]$ investigate high performance computing applied to cardiac electrophysiology to achieve quasi real-time simulations. Therefore, coupling a simulation of endovascular navigation with a model of cardiac electrophysiology while keeping performances close to real-time is extremely challenging.

Based on the Mentice VIST hardware (see Fig. 1(a)), we present a training system dedicated to interventional electrocardiology procedures that combines a biophysical modeling of cardiac electrophysiology with an endovascular catheterization. Based on our GPU model of cardiac electrophysiology, the training framework simulates the electro-anatomical mapping, perform RF ablation and electrical stimulation in real-time. Finally, a performance analysis is conducted based on a synthetic case of ventricular extrasystole.

\section{Material \& Methods}

\subsection{D-Image Based Model of the Heart}

The simulator is developed using $\mathrm{SOFA}^{1}$ and reproduces an intervention aiming at ablating an ectopic focus located in the RV. For a realistic navigation, the

\footnotetext{
${ }^{1}$ SOFA is an open source framework for interactive numerical simulations in medicine. More information about SOFA can be found at http://www. sofa-framework.org
} 


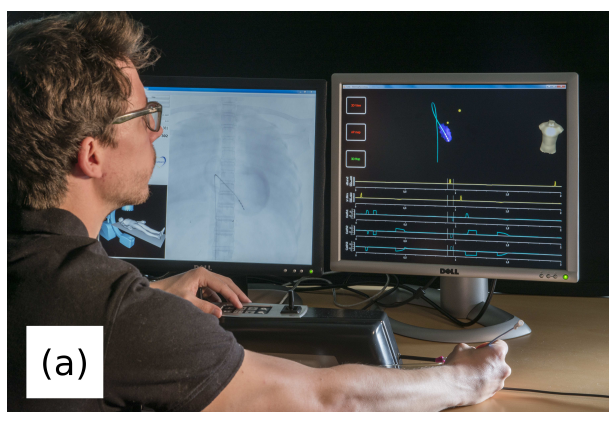

Fig. 1: (a) Our setup using the Mentice VIST device; (b) Resulting triangular mesh used cardiovascular navigation

cardiovascular anatomy is needed.Patient data were acquired in the framework of the European euHeart project ${ }^{2}$. 3D MRI are preoperatively obtained in order to reconstruct the patient-specific heart anatomy. Both atria and ventricles are labeled using a plugin tool implemented in GIMIAS ${ }^{3}$. The mask resulting from the segmented steady-state free precession magnetic resonance images (MRI) is then meshed using the CGAL library ${ }^{4}$. Dedicated to endovascular navigation, a first mesh generation of the four cardiac chambers is built including 12,950 triangular elements.A second, static and finer mesh, only modeling the ventricles, is extracted for the electrophysiology computation including 30,807 linear tetrahedra.

However, the catheter navigation simulation also requires a mesh of the venous system leading to the heart, since navigation starts from the femoral vein. Regarding blood vessels, synthetic data of the inferior vena cava are extracted from the Zygote data set ${ }^{5}$. The resulting mesh for navigation fuses the generic model of vena cava with the patient-specific mesh of the heart (presented in Fig. 1(b)).

After re-ordering the cardiac phases (from passive filling to ventricular isovolumetric relaxation), we estimate the cardiac motion from 4D cine MRI information using a Demon-based registration algorithm detailed in [8]. The estimated deformation field is resampled on the vertices of the navigation mesh, thus resulting in a realistic beating heart model.

\footnotetext{
${ }^{2}$ For more information about the euHeart project: www. euheart.eu

${ }^{3}$ GIMIAS is an open source framework providing image visualization, manipulation, and annotation. For more information: www.gimias .net

${ }^{4}$ CGAL is an open source software library that provides algorithms in computational geometry. For more information: www.cgal.org

${ }^{5}$ The Zygote data are a set of 3D anatomical models sold by the company Zygote Media Group. For more information: www.zygote.com
} 


\subsection{Catheter Navigation Model}

The real-time simulation of the catheter behavior during endovascular procedures is particularly challenging and has been the central interest of several research work. Catheters are wire-like structures characterized by stiff and light materials, high tensile strength and low resistance to bending. Our work relies on the FEM introduced by Duriez et al. in [6] and further developed by Dequidt et al. in [5], both in the context of coil embolization in neurology. This method is based on Kirchhoff rod theory, so that our catheter results in a serial set of beam elements. We choose this corotational approach since it handles geometric non-linearity due to large changes in the shape of the object. Other models exist for wire-like structures as the inextensible super-helices model proposed by Bertails et al. [2] or a linear representation of angular springs in Wang et al. [13]. However, super-helices from Bertails involve a quadratic time complexity regarding the number of helical elements. In comparison, corotational beam approach offers a linear complexity. Based on non-physical angular springs, the representation chosen by Wang implies non-physical behavior. The corotational model assumes that the strains remain "small" in a local frame defined at the level of each element. In our scope of endovascular navigation, the catheter undergoes large displacements but only small strains, which meets the corotational assumption.

The collision detection is performed using first a bounding volume hierarchy $(\mathrm{BVH})$ for the broad phase, and then computing local minimal distances, as introduced in [7]. Based on the Signorini's law combined with the Coulomb's law, the constraint resolution leads to a non-linear complementarity problem solved using a Gauss-Seidel algorithm.

\subsection{GPU Electrophysiology Model}

Our work is based on the Mitchell Schaeffer (MS) [10] model since $(i)$ it has only 5 parameters, (ii) each parameter has a physiological meaning and (iii) it provides a better estimation of the action potential compared to other phenomenological models (as the Aliev-Panfilov model [1]). Since it only captures the transmembrane potential $V_{m}$, the MS model is a "mono-domain" model. Only "bi-domain" models can simulate both intra-cellular $U_{i}$ and extra-cellular potentials $U_{e}$, where $V_{m}=U_{i}-U_{e}$.

To increase computation efficiency, the electrical activity of the heart is only simulated for the ventricles. This consideration is acceptable since atria and ventricles are electrically isolated by a collagen layer. In our model, the stimulation is induced by the Purkinje fibers. Relying on the finite element method, the ventricular electrophysiology is computed on a static mesh using 30,807 elements. Moreover, the implementation of the weak form of reaction diffusion equations leads to zero Neumann boundary conditions, i.e. the electrical current is null in the orthogonal direction of the border. Based on [12], the entire electrophysiology model is implemented on GPU. The simulation time step is constrained by 
the coarseness of the mesh. In this simulation, we use a full explicit backward differentiation integration scheme with a time step $d t_{E}=10^{-4} \mathrm{~s}$.

To integrate both electrophysiology and navigation simulations, we exploit the CPU parallelism and we choose a task scheduling architecture. This technique is an efficient way to scale the computation to all the CPU cores available. The main loop of our multithreading architecture is split into a parallel part executing concurrently the endovascular navigation and electrophysiology simulations and a serial part executing the graphics rendering. Electrophysiology simulation requires a very low time step $d t_{E} \leq 1.5 \cdot 10^{-4} \mathrm{~s}$ for stability reasons, whereas navigation simulation is running with $d t_{N} \leq 0.02 \mathrm{~s}$. Our framework can then be depicted as a asynchronous simulation based on multithreading.

\subsection{Interactive Model}

In the scope of ventricular extrasystole, the most time consuming step of the $\mathrm{RF}$ ablation procedure consists in locating and ablating the ectopic focus. This area includes excitable cells initiating a premature heart beat, or ectopic beat. Once catheters are positioned inside the heart cavities, the cardiologist needs to reconstruct an electro-anatomical mapping by acquiring activation times on the endocardium. The pathological area can be localized since the ectopic focus corresponds to the region with the earliest activation time. The complexity of this procedure highlights the importance of interacting with the electrophysiology in silico.

Extra-Cellular Potential Measurements During the procedure, cardiologists use catheters to interpret the electrical activity of the heart. These catheters can measure either unipolar or bipolar potentials, respectively measuring the extracellular potential $U_{e}$ or a difference of extra-cellular potentials $\Delta U_{e}$. Bipolar potentials are often preferred as they remove the far field potentials and provide sharper depolarizations. Since the MS model is mono-domain, the four-electrode catheter only records transmembrane potential (see Fig. 3(a)).

A realistic modeling of the displayed signals is key for our training simulator since cardiologists mainly rely on electrophysiological signals to understand the
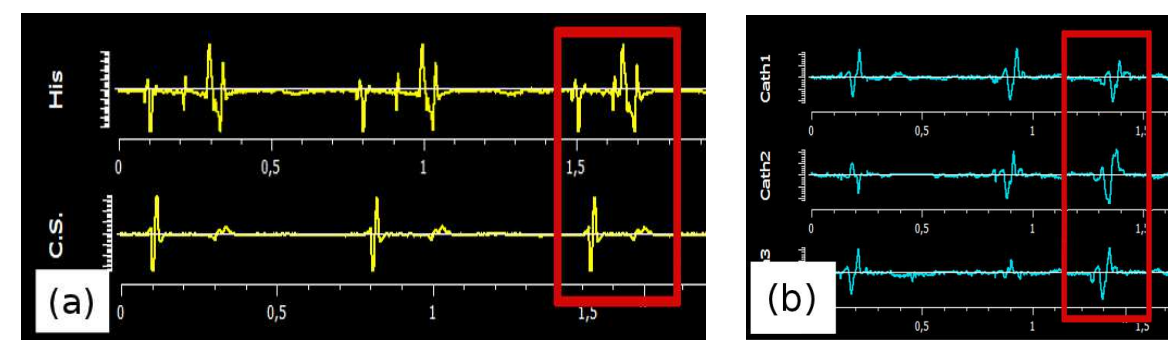

Fig. 2: (a) Unipolar measured at the His bundle and coronary sinus; (b) Bipolar signals recorded by the four electrodes 
cardiac arrhythmia and guide the ablation procedure. To reach this level of realism, real unipolar signals acquired at CHU Bordeaux are mapped on the simulated transmembrane potentials (see Fig. 2(a)). Each consecutive pair of electrodes thus computes one bipolar signal obtained by the difference of the two unipolar signals. The resulting bipolar signals acquired in silico are presented in Fig. 2(b). In the simulation, four electrodes are defined along the catheter tip and three bipolar signals driven by our MS model ("Cath1", "Cath2" and "Cath3") can be displayed.

Electro-Anatomical Mapping Once inside the targeted cardiac chamber, the electro-anatomical mapping starts in order to localize the arrhythmic substrate, here an ectopic focus. As with a real mapping system, the position of the catheter is tracked and the endocardium is partially reconstructed when the catheter touches the heart wall. In other words, as soon as a collision between the catheter and the endocardium occurs, the intersected triangles of the navigation mesh are displayed. Noise is added, so that the reconstructed surface looks realistic. The surface resulting from the virtual reconstruction is shown in Fig. 3(b).

After reconstructing the endocardium, the simulation allows to build a map of activation times based on the extra-cellular measurements. When an ectopic stimulation starts while mapping, our framework computes local activation times, which are mapped on the endocardial surface. The activation times correspond to the elapsed time between the depolarization of the ectopic focus and the depolarization of the point currently in contact with the catheter. By measuring the extrasystolic activation times on the endocardium, the cardiology trainee can thus determine the exact location of the arrhythmia. The Fig. 3(c) captures this electro-anatomical mapping simulated during our virtual procedure.

RF Ablation Once a target region has been identified, the cardiologist performs the RF ablation by heating the tissue using a RF (usually from 300 to $700 \mathrm{kHz}$ ) alternating current. This energy is delivered through an electrode in contact with the target tissue. When the temperature exceeds $60^{\circ} \mathrm{C}$, denaturing of proteins
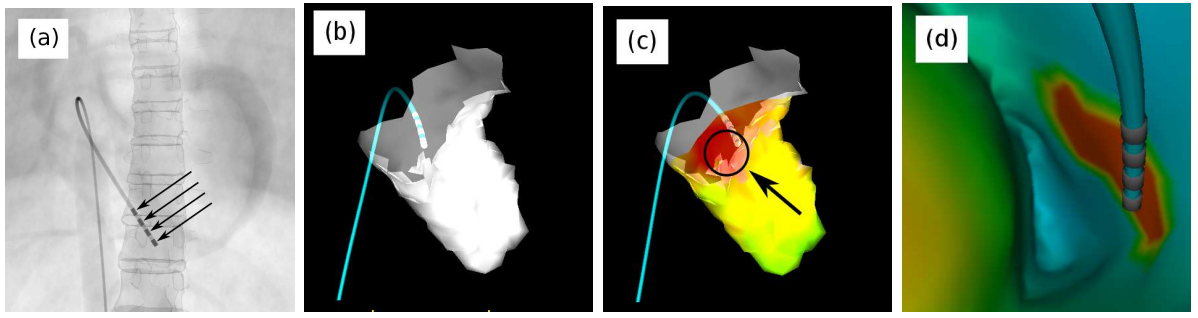

Fig. 3: (a) Fluoroscopic view of four electrodes (arrows) at the distal extremity of the catheter; (b) Reconstruction of the 3D RV endocardium; (c) Extrasystolic activation times with the ectopic focus (arrow); (d) Internal view of the RV where the red area underwent ablation 
leads to a cellular death with coagulation necrosis. To be efficient, the temperature must nevertheless not exceed $100^{\circ} \mathrm{C}$. After ablation, cardiac cells lose their electrical conductivity. As a consequence, a successful procedure assumes that the regions originally responsible for the electrical disorder are well electrically isolated.

In our simulation, the ablation step is modeled by a progressive decrease of the electrical conductivity inside the tetrahedra. A zero conductivity is associated to dead cardiac cells. The effect of ablation gradually propagates from edge to edge through the myocardium. The longer the ablation, the larger the ablation area. Our training simulator also allows to set the desired power of ablation, thus making the ablation process faster or slower. During the simulation, the ablation is triggered by one of both pedals provided with the tracking device. The tissue electrical conductivity is consequently updated in real-time. An illustration of an ablation scar is given in Fig. 3(d).

Stimulation A stimulation can be used by the cardiologist to assess the success of the ablation procedure. When the catheter touches the heart wall, an electrical current can be delivered in the region of contact. Moreover, the current value of this catheter stimulus $J_{\text {cath-stim }}(t)$ is interactively set by the user. As for the ablation, the stimulation is triggered by the user using the pedals of the Mentice device. If no abnormal activity is detected during the stimulation process, the arrhythmic substrate is successfully ablated.

\section{Results}

Exploiting the power of both multithreading and GPU computing, we achieve a fully interactive simulation. Our simulation runs on a computer including an Intel Core i7 CPU and an NVidia GTX 580 GPU. The performance results are given in Table 1 regarding the different steps during the procedure. In this table, realtime ratio stands for the ratio of elapsed time over computation time. When the simulation is faster than real-time (ratio $>1$ ), the computation can be slowed

\begin{tabular}{|c|c|c|c|c|}
\hline \multicolumn{2}{|c|}{} & \multicolumn{2}{|c|}{ Training Simulator } \\
\hline Position & Number of contacts & $\begin{array}{c}\text { Frames } \\
\text { per second }\end{array}$ & $\begin{array}{c}\text { Real-time } \\
\text { ratio }\end{array}$ \\
\cline { 2 - 4 } Start & 0 & {$[0-0]$} & 44.0 & 0.88 \\
\hline Vena cava & 2 & {$[2-2]$} & 43.8 & 0.87 \\
\hline $\begin{array}{c}\text { Entrance } \\
\text { of atrium }\end{array}$ & 5 & {$[2-14]$} & 31.6 & 0.63 \\
\hline Final & 50 & {$[41-65]$} & 23.6 & 0.47 \\
\hline
\end{tabular}

Table 1: Performance results of the whole training framework 
down to retain real-time. From Table 1, we first realize that the computational efficiency is strongly related to the number of contacts, i.e. to the navigation part. At the beginning of the procedure, only few contacts are detected whereas, once inside the heart, the catheter leans against the endocardium, thus decreasing the performance. This reveals that the navigation part tends to limit the overall performance when many contacts (over 50) are detected. 50 contacts implies 150 constraints which is substantial regarding the number of beams in our model (40 beams).

\section{Conclusion}

Table 1 shows that our training system runs between 1.1 and 2.3 times slower than real-time. Our hybrid architecture proves to be efficient so that the entire simulation remains interactive. Another crucial feature regarding our scope is that the interactions with the electrophysiology model do not affect the performances. As a consequence, this training simulator for cardiac RF ablation procedure already offers close to real-time performances and a high level of interactivity. Finally, a clinical evaluation is currently being conducted to assess the realism of our virtual training. This degree of validation would enlarge the field of application of our simulator.

\section{References}

1. Aliev, R., Panfilov, A.: A simple two-variable model of cardiac excitation. Chaos, Solitons and Fractals 7(3), 293-301 (1996)

2. Bertails, F., Audoly, B., Cani, M.P., Querleux, B., Leroy, F., Lévêque, J.L.: Superhelices for predicting the dynamics of natural hair. In: ACM Transactions on Graphics. vol. 25, pp. 1180-1187 (2006)

3. Chiang, P., Zheng, J., Yu, Y., Mak, K., Chui, C., Cai, Y.: A vr simulator for intracardiac intervention. Computer Graphics and Applications 33(1), 44-57 (2013)

4. Dawson, S., Cotin, S., Meglan, D., Shaffer, D., Ferrell, M.: Designing a computerbased simulator for interventional cardiology training. Catheterization and Cardiovascular Interventions 51(4), 522-527 (2000)

5. Dequidt, J., Marchal, M., Duriez, C., Kerien, E., Cotin, S.: Interactive simulation of embolization coils: Modeling and experimental validation. In: MICCAI 2008. vol. 5241, pp. 695-702. Springer (2008)

6. Duriez, C., Cotin, S., Lenoir, J., Neumann, P.: New approaches to catheter navigation for interventional radiology simulation 1. Computer Aided Surgery 11(6), 300-308 (2006)

7. Johnson, D., Willemsen, P.: Accelerated haptic rendering of polygonal models through local descent. In: Haptic Interfaces for Virtual Environment and Teleoperator Systems, 2004. pp. 18-23 (2004)

8. Mansi, T., Pennec, X., Sermesant, M., Delingette, H., Ayache, N.: iLogDemons: A demons-based registration algorithm for tracking incompressible elastic biological tissues. International Journal of Computer Vision 92(1), 92-111 (2011)

9. Maron, B.J., Towbin, J.A., Thiene, G., Antzelevitch, C., Corrado, D., Arnett, D., Moss, A.J., Seidman, C.E., Young, J.B.: Contemporary definitions and classification of the cardiomyopathies. Circulation 113(14), 1807-1816 (2006) 
10. Mitchell, C., Schaeffer, D.: A two-current model for the dynamics of cardiac membrane. Bulletin of Mathematical Biology 65, 767-793 (2003)

11. Rapaka, S., Mansi, T., Georgescu, B., Pop, M., Wright, G., Kamen, A., Comaniciu, D.: Lbm-ep: Lattice-boltzmann method for fast cardiac electrophysiology simulation from 3d images. In: MICCAI 2012. vol. 7511, pp. 33-40. Springer (2012)

12. Talbot, H., Marchesseau, S., Duriez, C., Sermesant, M., Cotin, S., Delingette, H.: Towards an interactive electromechanical model of the heart. Journal of the Royal Society Interface Focus 3(2) (April 2013)

13. Wang, F., Duratti, L., Samur, E., Spaelter, U., Bleuler, H.: A computer-based real-time simulation of interventional radiology. In: Engineering in Medicine and Biology Society, 2007. pp. 1742-1745. IEEE (2007) 